\title{
Contact Physics Modeling and Optimization Design of RF-MEMS Cantilever Switches
}

\author{
Z. Wang, L. Chow, J. L. Volakis, K. Saitou and K. Kurabayashi
}

\begin{abstract}
RF MEMS direct-contact switches exhibit many advantages over the conventional semiconductor switches; however, existing drawbacks such as low power handling, high pull-in voltage and long switch opening time are most critical. This paper presents an optimization design for RF-MEMS cantilever direct-contact switch to achieve maximum power handling capability, minimum pull-in voltage and switch opening time simultaneously. A 2-step optimization technique is proposed to achieve the optimal design to allow for a power handling capability of $130 \mathrm{~mW}$, a pull-in voltage of $52 \mathrm{~V}$, and a switch opening time $4.4 \mu$ s presented. The optimization results show that substantial room exists for improving the current designs of RF MEMS direct-contact switches
\end{abstract}

\section{Introduction}

Surface micromachining techniques have generated a new class of RF switches that exhibit ultra-low loss and high linearity [1]. As an example, MEMS direct-contact switches (Fig. 1) can achieve less than $0.1 \mathrm{~dB}$ loss from DC up to $40 \mathrm{GHz}$ and this is attractive for radar and communications antennas [2] as well as tunable filters [3]. However, RF MEMS contact switches suffer from high actuation voltage, low power handling capability, and long switch opening time [8]. Chan [4] and Goldsmith [5] mention that high actuation voltage may lead to shorter lifetime for MEMS switches. Schauwecker, et al [6] and Rebeiz [7] mention that the maximum input power for RFMEMS contact switches should be lower than $100 \mathrm{~mW}$, but the requirements for most wireless applications are much more demanding. Therefore, it is of significant engineering interest to improve the actuation voltage, power handling capability and switch opening speed.

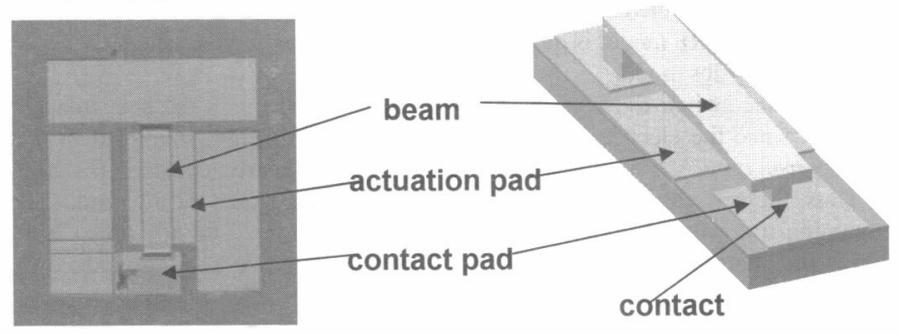

Fig.1 Optical image and a schematic (right) of a RF MEMS cantilever direct switch.

This paper addresses the optimization of cantilever RF-MEMS direct-contact switches for improved power handling, actuation voltage, and switching speed. We carry out the optimization design based on multi-physics (electromagnetic, electrostatic thermal, and structural) modeling and optimization techniques [9]. Specifically, an embedded contact physics model was developed to calculate the contact temperature for RF MEMS switches at the down-state based on the maximum RF power without causing contact softening [10]. Separately, an integrated electrostatic/structural model with an embedded adhesion model [11] is proposed to calculate the pull-in voltage and switch restoring force to determine the switch opening time. By coupling these physical 
models with optimization algorithm we are able to determine the in-plane switch geometry, dimple size and electrical resistance of the contact surfaces subject to 1) maximum input power, 2) minimum actuation voltage, and 3) maximum switch opening speed.

The sections below describe an electromagnetic/thermal contact physics model (Section II); our 2-step optimization design scheme and an example of optimization (Part III).

\section{Electromagnetic/Thermal Contact Physics Model}

Electromagnetic contact-physics model

The electromagnetic (EM) modeling of RF MEMS switches is a challenging task due to their extremely small size. An extended finite-element boundary-integral (EFE-BI) for EM modeling of the up-state of RF MEMS switches [9] was specifically developed for their analysis. For the down state, the contact physics modeling is more readily realizable. This is facilitated by modeling the contact asperities using an equivalent resistive sheet as shown in Fig. 2.

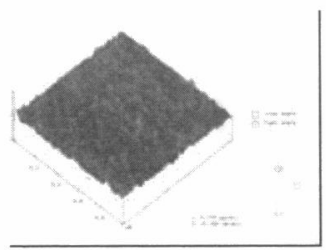

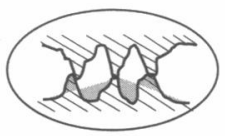

(b)

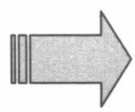

(c)

(a)

Fig. 2 (a) Three dimensional AFM image of the gold surface at switch contact; (b) 2D contact surface element; (c) equivalent contact model using a resistive sheet

The impedance/resistivity for the contact-area elements are represented by a parallelconnected equivalent contact resistance and capacitance $C$ distributed to each finite element within the finite element domain. In conjunction with the EFIE-BI [9], we found that the realized RF model is quite accurate in predicting joule heating.

Thermal contact-physics model

Current flow in the switch causes joule heating in accordance with the heat transfer equation 9]. Since the electrical signal period $(1 / \mathrm{f})$ is sufficiently smaller than the thermal response time, the electrical dissipation appears nearly constant for heat transfer. Thus, the rms value of the current density calculated with the EM model is used (assuming a constant temperature at each re-calculation). The additional heat generated at the contact due to the electrical contact resistance $R_{c}$ is simply added to the contacting elements.

\section{Optimal designs of cantilever RF MEMS switches}

Upon embedding, the above contact-physics models into the EFE-BI $[9,11]$, the optimization of the cantilever RF-MEMS switches was carried out to improve power handling, actuation voltage, and switching speed. To start with, a sensitivity study of the beam's dimensions, dimple size and contact resistance's effect on the power handling capability are illustrated in Fig.3 (referring to a typical beam with $200 \times 100 \times 7 \mu \mathrm{m}$, dimple size $10 \mu \mathrm{m}^{2}$, contact resistance $1.5 \Omega$ and contact roughness $20 \mathrm{~nm}$ ). From the pie chart, we note that the contact resistance and dimple size are dominant on the power handling capability, whereas the beam dimensions and effect on power handling capability may be negligible. Taking the fact that the dimple size has little or no effect on the pull-in voltage and restoring force, the original (more 
complex) optimization problem may be separated into a 2 -step, relatively simple, process:

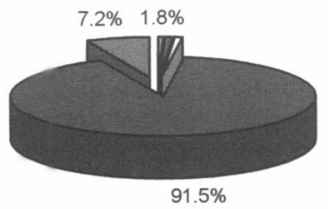

QLength (100 250 um)
$\square$ Width (50 200 um)
$\square$ Thickness $(4 \sim 10 \mathrm{um})$
Contact resistance $(1 \sim 2$ Ohm $)$
$\square$ Contact area $\left(4 \sim 16 \mathrm{um}^{\wedge} 2\right)$

Fig.3 Power handling capability factors

I. Dimple size, restoring force and contact resistance optimization to achieve maximum power handling capability with short opening time.

II. Beam dimension \& shape optimization to achieve minimum pull-in voltage with constraint on the required restoring force from optimization I.

A typical switch $(200 \times 100 \times 7 \mu \mathrm{m})$ is used to show the 2-step optimization approach.

Optimization I Dimple size and contact resistance optimization

The dimple size, contact resistance, and restoring force determine the power handling capability and opening time. The objectives of this optimization are to minimize the switch opening time and concurrently maximize its power handling by varying the dimple size $\left(1 \sim 16 \mu \mathrm{m}^{2}\right)$, dimple (larger than the skin depth), contact resistance (1 3 $\Omega$ ), and restoring force $(25 \sim 60 \mu \mathrm{N})$, while maintaining a temperature range of $60^{\circ}$ $80^{\circ} \mathrm{C}$ (constraint).

Fig.4 (a) shows the optimized Pareto-curve for the power handling capability vs. opening time. To achieve the maximum power handling capability and short opening time, the optimized restoring force was found to be $40 \mu \mathrm{N}$ (by optimization II). Also, the optimized dimple size was found to be $4.65 \mu \mathrm{m}^{2}$ with a width of $1.70 \mu \mathrm{m}$. Further the optimized contact resistance was $1.5 \Omega$.

\section{Optimization II Beam shape and dimension optimization}

As well-known, an optimized beam shape may achieve the minimum pull-in voltage while maintaining the required switch restoring force. A cantilever beam of length $200 \mu \mathrm{m}$ and thickness $7 \mu \mathrm{m}$ with an actuation pad $140 \mu \mathrm{m}$ wide are used for this optimization for the SQP technique. To obtain the global optimization result, an 'error-and-try' process was utilized with different initial values to reach the 'global' optimum result. Here, the beam length $(200 \mu \mathrm{m})$, thickness $(7 \mu \mathrm{m})$, and its gap $(1.7$ $\mu \mathrm{m})$ are fixed.

The problem is defined by minimizing the pull-in voltage with the beam width as a variable and the restoring force set to be $40 \mu \mathrm{N}$ (Optimization I). Fig.4 (b) provides the optimized beam shape for a pull-in voltage of $57.64 \mathrm{~V}$.

Combining the above two optimization processes, the overall objectives are listed in table I. It is clear that the optimized beam and dimple can achieve better switch performance as compared to typical values reported so far.

Table I Comparison of Typical value [8] vs. our optimized value

\begin{tabular}{|c|c|c|c|}
\hline & Opening time & Power handling & Pull-in voltage \\
\hline Typical value & $5 \mu \mathrm{s}-200 \mathrm{~ms}$ & $20-50 \mathrm{~mW}$ & $50-80 \mathrm{~V}$ \\
\hline $\begin{array}{c}\text { Our optimized } \\
\text { value }\end{array}$ & $4.4 \mu \mathrm{s}$ & $130 \mathrm{~mW}$ & $57 \mathrm{~V}$ \\
\hline
\end{tabular}




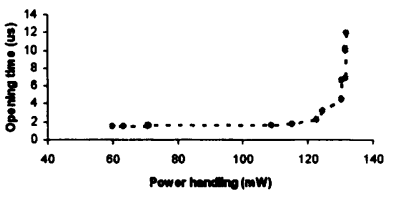

(a)

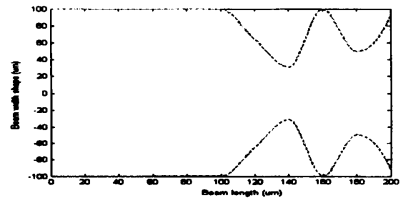

(b)

Fig. 4 (a) Pareto curve of power handling vs. opening time; (b) Optimization beam shape (top view) for minimum pull-in voltage with a restoring force set at $40 \mu \mathrm{N}$

\section{Conclusion}

Our study found that RF MEMS switch design benefit directly from optimization. Here simple 2-step optimization scheme was used and found sufficient to design.: 1) The beam shape and dimensions leading to low pull-in voltage and large restoring force; 2) the optimum dimple dimensions, contact resistance and restoring force to achieve high power handling and short opening time. These two optimization steps were then coupled by the restoring force. Our optimization design example demonstrates that substantial room exists to achieve better switch performance.

\section{References}

[1] C. Goldsmith, Z. Yao, S.Eshelman, and D. Denniston, "Performance of low-loss RF EMMS capacitive siwthces," IEEE Microwave and Guided Wave Lett., Vol.8, no.8, pp.269-271, Aug. 1998.

[2] B.R. Norvell, R.J. Hancock, J.K. Smith, M.L. Pugh, S.W. Theis, and J. Kviatkofsky, "Microelectro mechanical switch (MEMS) technology applied to electronically scanned arrays for space based radar," Proc. 1999 Aerospace Conference, Vol.3, pp.239-247, 1999

[3] C.L. Goldmith, A. Malczewski, Z.J. Yao, S. Chen, J. Ehmke, and D.H. Hinzel, "RF MEMS Variable Capacitors for Tunable Filters," Int. J. of $R F$ and Microwave CAE, v0l.9, no.4, pp.362-374, July 1999.

[4] R. Chan, R. Lesnick, D. Becher, and M. Feng, "Low-actuation voltage RF MEMS shunt switch with cold switching lifetime of seven billion cycles," $J$. of Microelectromechanical Systems, vol.12, no.5, pp.713-719, 2003.

[5] C. Goldsmith, J. Ehmke, A. Malczewski, B. Pillans, S. Eshelman, Z. Yao, J. Brank, and M. Eberly, "Lifetime characterization of capacitive RF MEMS switches", IEEEMTT-S TU3C-5, pp.227-230, 2001

[6] B. Schauwecker, K.M. Strohm, W. Simon, J. Mehner, and J.-F. Luy, "A new type of high bandwidth RF MEMS Switch-toggle switch," J. of Semiconductor Technology and Science, vol.2, no.4, pp.237-245, Dec. 2002.

[7] G.M. Rebeiz and J.B. Muldavin, "RF MEMS switches and switch circuits", IEEE Microwave Magazine, vol.2, pp.59-71, Dec.2001.

[8] G. M. Rebeiz, RF MEMS- Theory, Design, and Technology, Wiley 2002

[9] Wang, Z., Jensen, B., Volakis, J.L., Saitou, K., and Kurabayashi, K., "Full-wave electromagnetic and thermal modeling for prediction of heat-dissipation-induced RF-MEMS switch Failure," IEEE J. MEMS, submitted for review

[10] B. D. Jensen, K. Huang, L. L.-W. Chow and K. Kurabayashi, Low-force contact heating and softening using micromechanical switches in diffusive-ballistic electron transport transition, J. of Physics Lett., submitted for review.

[11] B.D. Jensen, L.-W. Chow, John L. Volakis, and Katsuo Kurabayashi, "Adhesion Effects on Contact Opening Time in MEMS Switches," in Proc. 2004 ASME/STLE International Joint Tribology 